\title{
Synthesis and Anticancer Activities of 4-[(Halophenyl)diazenyl]phenol and 4-[(Halophenyl)diazenyl]phenyl Aspirinate Derivatives against Nasopharyngeal Cancer Cell Lines
}

\author{
Boon Kui Ho, ${ }^{1}$ Zainab Ngaini, ${ }^{1}$ Paul Matthew Neilsen, ${ }^{2,3}$ Siaw San Hwang, ${ }^{2}$ \\ Reagan Entigu Linton, ${ }^{2}$ Ee Ling Kong, ${ }^{2}$ and Boon Kiat Lee ${ }^{2}$ \\ ${ }^{1}$ Department of Chemistry, Faculty of Resource Science and Technology, Universiti Malaysia Sarawak, 94300 Kota \\ Samarahan, Sarawak, Malaysia \\ ${ }^{2}$ Cancer Research and Advanced Therapeutic Group (CREATE), Faculty of Engineering, Computing and Science, \\ Swinburne University of Technology Sarawak Campus, Jalan Simpang Tiga, 93350 Kuching, Sarawak, Malaysia \\ ${ }^{3}$ School of Health, Medical and Applied Sciences, Central Queensland University, North Rockhampton, QLD 4702, Australia
}

Correspondence should be addressed to Zainab Ngaini; nzainab@unimas.my

Received 15 February 2017; Accepted 18 June 2017; Published 25 July 2017

Academic Editor: Liviu Mitu

Copyright (C) 2017 Boon Kui Ho et al. This is an open access article distributed under the Creative Commons Attribution License, which permits unrestricted use, distribution, and reproduction in any medium, provided the original work is properly cited.

\begin{abstract}
Aspirin and azo derivatives have been widely studied and have drawn considerable attention due to diverse biological activities. In this study, a series of 4-[(halophenyl)diazenyl]phenyl aspirinate derivatives were synthesized from the reaction of aspirin with 4-[(halophenyl)diazenyl]phenol via esterification, in the presence of DCC/DMAP in DCM with overall yield of 45-54\%. 4[(Halophenyl)diazenyl] phenol was prepared prior to esterification from coupling reaction of aniline derivatives and phenol in basic solution. All compounds were characterized using elemental analysis, FTIR, and ${ }^{1} \mathrm{H}$ and ${ }^{13} \mathrm{C}$ NMR spectroscopies. All compounds were screened for their anticancer activities against nasopharyngeal cancer (NPC) HK-1 cell lines and the viability of cultured cells was determined by MTS [3-(4,5-dimethylthiazol-2-yl)-5-(3-carboxylmethoxylphenyl)-2-(4-sulfophenyl)-2H-tetrazolium]based colorimetric assay. 4-[(E)-(Fluorophenyl)diazenyl]phenol showed the highest anticancer activity against NPC HK-1 cell lines compared to other synthesized compounds. 4-[(Halophenyl)diazenyl]phenyl aspirinate showed low cytotoxicity against NPC HK-1 cell lines compared to 4-[(halophenyl)diazenyl]phenol but better anticancer activity than aspirin alone.
\end{abstract}

\section{Introduction}

Cancer has become a serious clinical problem worldwide [1]. It is because cancer cells have the ability to grow rapidly and develop into malignancy tumor [2]. Nasopharyngeal cancer is the most common malignant tumor and occurs in the nasopharynx, the upper part of the throat near to the neck [3]. Nasopharyngeal cancer (NPC) is commonly developed from infection with Epstein-Barr virus, foods, and family history [4]. NPC, which also known as HK-1, was found to have high level of epidermal growth factor receptor (EGFR) protein [5]. Hence, by targeting the EGFR protein, it might become a new therapeutic strategy to inhibit the HK-1 cells [6]. The usage of drugs could stop the growth of the malignant cells in one's body by inhibiting its EGFR pathway of the cancer cells itself [7].

The development of new drug for cancer treatment has gained much interest in recent years. The modification of pharmacologically active compounds may lead to a new finding of novel drugs with diverse pharmacological activities [8]. Chemical modification of aspirin, for instance, has been reported to reduce gastrointestinal toxicity and exhibited diverse biological activities such as anticancer [9], antiinflammatory [10], and antibacterial $[11,12]$ activities. Aspirin 
bearing active functional groups such as diethyl phosphate and nitro groups has been reported to have anticancer activity against pancreatic and colon cancers $[13,14]$.

Azo is another active compound reported for its pharmaceutical importance as antidiabetic [15], antineoplastic [16], antibacterial [17], and anticancer [18] agent. Azo molecules are known to be involved in the inhibition of DNA, RNA, carcinogenesis, and protein synthesis [19]. The presence of $-\mathrm{N}=\mathrm{N}$ - in the molecular structure of azo is responsible for the interaction with the active site of protein [20]. The presence of halogen substituents in azo molecule also played an important role in the inhibition of receptor enzyme [21].

In this study, a series of azo derivatives (1a-d) and aspirin azo derivatives (2a-d) have been synthesized and demonstrated for potential anticancer agent against nasopharyngeal cancer cell lines. The viability of the cultured cells was determined by MTS-based colorimetric assay and discussed.

\section{Materials and Methods}

2.1. Materials. Chemical agents used in the present study included dimethyl sulfoxide (DMSO, Sigma-Aldrich), penicillin-streptomycin (GIBCO), fetal bovine serum (FBS, SigmaAldrich), and RPM1 1640 (GIBCO).

2.2. Characterizations. Melting point was measured by Stuart SMP3 melting point apparatus and the elemental analysis was determined by flash EA1112 analyzer. IR spectra $\left(v / \mathrm{cm}^{-1}\right)$ were recorded on a Perkin Elmer GX spectrophotometer able to show infrared spectra of functional groups, potassium bromide pellet $(\mathrm{KBr})$ solid material. ${ }^{1} \mathrm{H}$ NMR spectra were recorded on a JEOL.ECA 500 spectrometer at $500 \mathrm{MHz}$ and ${ }^{13} \mathrm{C} \mathrm{NMR}$ at $125 \mathrm{MHz}$. Tetramethylsilance (TMS) was used as a reference for both spectra while $\mathrm{CDCl}_{3}$ and $\mathrm{DMSO}-\mathrm{d}_{6}$ were used as solvents.

2.3. General Method for the Synthesis of Halogenated Azo Compounds $\mathbf{1 a}-\boldsymbol{d}$. A mixture of 4-fluoroaniline $(0.56 \mathrm{~g}$, $5 \mathrm{mmol})$ and $\mathrm{HCl}(2 \mathrm{M}, 6 \mathrm{~mL})$ was added slowly to $\mathrm{NaNO}_{2}$ in water $(1 \mathrm{M}, 2 \mathrm{~mL})$ at $0-5^{\circ} \mathrm{C}$. Phenol $(0.47 \mathrm{~g}, 5 \mathrm{mmol})$ was dissolved in $\mathrm{NaOH}(2 \mathrm{M}, 10 \mathrm{~mL})$ and cooled to $0-5^{\circ} \mathrm{C}$. The diazo salt was added slowly to phenol solution at $0-5^{\circ} \mathrm{C}$ and stirred for $40 \mathrm{~min}$. $\mathrm{HCl}(2 \mathrm{M}, 10 \mathrm{~mL})$ was added to form precipitate and filtered and washed with cold water $(2 \times 20 \mathrm{~mL})$. The crude product was recrystallized from hot ethanol to give (1a) $(0.56 \mathrm{~g}, 52 \%)$ as purple solid; m.p. $158-159^{\circ} \mathrm{C}$. (Found: C, 66.58; H, 4.12; N, 12.80\% $\mathrm{C}_{12} \mathrm{H}_{9} \mathrm{~N}_{2}$ OF Requires C 66.67; $\mathrm{H}, 4.17 ; \mathrm{N}, 12.96 \%) ; R_{f} 0.54$ (1:4 EA/hexane) IR: $v_{\max }$ (thin film $\left./ \mathrm{cm}^{-1}\right) 3135(\mathrm{OH}), 1591(\mathrm{C}=\mathrm{C}$ aromatic), $1471(\mathrm{~N}=\mathrm{N}), 1235$ (C-N), 1102 (C-O), 839 (C-F) ${ }^{1} \mathrm{H}$ NMR (500 MHz, DMSO$\left.\mathrm{d}_{6}\right) \operatorname{ppm} 6.94\left(2 \mathrm{H}, \mathrm{d}, J=6.85 \mathrm{~Hz}, \operatorname{Ar}-\mathrm{H}_{3^{\prime}}\right) 7.37(2 \mathrm{H}, \mathrm{d}, J=$ $\left.8.78 \mathrm{~Hz}, \mathrm{Ar}-\mathrm{H}_{3}\right)$ 7.78-7.88 (4H, m, Ar- $\left.\mathrm{H}_{2,2^{\prime}}\right) 10.32(1 \mathrm{H}, \mathrm{s}, \mathrm{OH})$ ${ }^{13} \mathrm{C} \mathrm{NMR}\left(125 \mathrm{MHz}, \mathrm{CDCl}_{3}\right) 116.00\left(\mathrm{Ar}-\mathrm{C}_{3}\right), 116.21\left(\mathrm{Ar}^{-\mathrm{C}_{3^{\prime}}}\right)$, $124.58\left(\mathrm{Ar}^{-\mathrm{C}_{2}}\right), 124.65\left(\mathrm{Ar}-\mathrm{C}_{2}\right), 125.13\left(\mathrm{Ar}-\mathrm{C}_{1^{\prime}}\right), 147.02(\mathrm{Ar}-$ $\left.\mathrm{C}_{1}\right), 149.32\left(\mathrm{Ar}-\mathrm{C}_{4^{\prime}}\right), 158.63\left(\mathrm{Ar}-\mathrm{C}_{4}\right)$. The others, $\mathbf{1 b}-\mathbf{d}$, were synthesized by repeating the same procedure as $\mathbf{1 a}$.

4-[(E)-(Chlorophenyl)diazenyl]phenol (1b) (0.62 g, 53\%) as yellow solid; m.p. $154-155^{\circ}$ C. (Found: C, 61.68; H, 3.86; N,
$11.98 \% \mathrm{C}_{12} \mathrm{H}_{9} \mathrm{~N}_{2} \mathrm{OCl}$ Requires C, 61.94; H, 3.87; N, 12.04\%); $R_{f} 0.57$ (1:4 EA/hexane) IR: $v_{\max }\left(\right.$ thin film $\left./ \mathrm{cm}^{-1}\right) 3186(\mathrm{OH})$, $1587(\mathrm{C}=\mathrm{C}$ aromatic), $1474(\mathrm{~N}=\mathrm{N}), 1221(\mathrm{C}-\mathrm{N}), 1080(\mathrm{C}-\mathrm{O})$, 836 (C-Cl). ${ }^{1} \mathrm{H}$ NMR $\left(500 \mathrm{MHz}, \mathrm{CDCl}_{3}\right)$ ppm $5.46(1 \mathrm{H}, \mathrm{s}$, $\mathrm{OH}) 6.96\left(2 \mathrm{H}, \mathrm{d}, J=8 \mathrm{~Hz}, \mathrm{Ar}-\mathrm{H}_{3^{\prime}}\right) 7.46(2 \mathrm{H}, \mathrm{d}, J=8 \mathrm{~Hz}, \mathrm{Ar}-$ $\left.\mathrm{H}_{2^{\prime}}\right) 7.82\left(2 \mathrm{H}, \mathrm{d}, J=8 \mathrm{~Hz}, \mathrm{Ar}-\mathrm{H}_{2}\right) 7.86(2 \mathrm{H}, \mathrm{d}, J=8 \mathrm{~Hz}, \mathrm{Ar}-$ $\left.\mathrm{H}_{3}\right){ }^{13} \mathrm{C} \mathrm{NMR}\left(125 \mathrm{MHz}, \mathrm{CDCl}_{3}\right) 116.0\left(\mathrm{Ar}_{-} \mathrm{C}_{3^{\prime}}\right), 123.98(\mathrm{Ar}-$ $\left.\mathrm{C}_{2^{\prime}}\right), 125.25\left(\mathrm{Ar}-\mathrm{C}_{2}\right), 129.42\left(\mathrm{Ar}-\mathrm{C}_{3}\right), 136.46\left(\mathrm{Ar}-\mathrm{C}_{4}\right), 147.17$ $\left(\right.$ Ar- $\left.\mathrm{C}_{1^{\prime}}\right), 151.42\left(\right.$ Ar- $\left.\mathrm{C}_{1}\right), 158.76\left(\right.$ Ar- $\left.\mathrm{C}_{4^{\prime}}\right)$.

4-[(E)-(Bromophenyl)diazenyl]phenol (1c) (0.89 g, 64\%) as red solid; m.p. $165-166^{\circ} \mathrm{C}$. (Found: C, 51.20; H, 3.16; N, $10.05 \% \mathrm{C}_{12} \mathrm{H}_{9} \mathrm{~N}_{2} \mathrm{OBr}$ Requires C, 51.99; $\left.\mathrm{H}, 3.25 ; \mathrm{N}, 10.11 \%\right) ; R_{f}$ 0.56 (1: 4 EA/hexane) IR: $v_{\max }\left(\right.$ thin film $\left./ \mathrm{cm}^{-1}\right) 3076(\mathrm{OH})$, 1588 (C=C aromatic), $1473(\mathrm{~N}=\mathrm{N}), 1225$ (C-N), 1101 (C-O), 833 (C-Br). ${ }^{1} \mathrm{H}$ NMR $\left(500 \mathrm{MHz}, \mathrm{CDCl}_{3}\right)$ ppm $5.54(1 \mathrm{H}, \mathrm{s}$, $\mathrm{OH}) 6.94\left(2 \mathrm{H}, \mathrm{d}, J=8.6 \mathrm{~Hz}, \mathrm{Ar}-\mathrm{H}_{3^{\prime}}\right) 7.62(2 \mathrm{H}, \mathrm{d}, J=8.6 \mathrm{~Hz}$ Ar- $\left.\mathrm{H}_{2^{\prime}}\right) 7.74\left(2 \mathrm{H}, \mathrm{d}, J=8.6 \mathrm{~Hz}, \operatorname{Ar}-\mathrm{H}_{2}\right) 7.86(2 \mathrm{H}, \mathrm{d}, J=$ $\left.8.6 \mathrm{~Hz}, \mathrm{Ar}-\mathrm{H}_{3}\right) .{ }^{13} \mathrm{C} \mathrm{NMR}\left(125 \mathrm{MHz}, \mathrm{CDCl}_{3}\right)$ ppm $116.02(\mathrm{Ar}-$ $\left.\mathrm{C}_{3^{\prime}}\right), 124.23\left(\mathrm{Ar}-\mathrm{C}_{2^{\prime}}\right), 124.79\left(\mathrm{Ar}-\mathrm{C}_{4}\right), 125.28\left(\mathrm{Ar}-\mathrm{C}_{2}\right), 132.39$ $\left(\right.$ Ar- $\left.\mathrm{C}_{3}\right), 147.10\left(\right.$ Ar-C $\left._{1^{\prime}}\right), 151.48\left(\right.$ Ar- $\left.C_{1}\right), 158.76\left(\right.$ Ar-C $\left._{4^{\prime}}\right)$.

4-[(E)-(Iodophenyl)diazenyl]phenol (1d) $(0.68 \mathrm{~g}, 42 \%)$ as purple solid; m.p. $168-170^{\circ} \mathrm{C}$. (Found: C, 44.06; H, 2.76; N, $8.44 \% \mathrm{C}_{12} \mathrm{H}_{9} \mathrm{~N}_{2}$ OI Requires C, 44.44; $\left.\mathrm{H}, 2.78 ; \mathrm{N}, 8.64 \%\right) ; R_{f}$ 0.54 (1: 4 EA/hexane) IR: $v_{\max }\left(\right.$ thin film $\left./ \mathrm{cm}^{-1}\right) 3172(\mathrm{OH})$, $1591(\mathrm{C}=\mathrm{C}$ aromatic), $1471(\mathrm{~N}=\mathrm{N}), 1242(\mathrm{C}-\mathrm{N}), 1074(\mathrm{C}-\mathrm{O})$, $837(\mathrm{C}-\mathrm{I}){ }^{1} \mathrm{H}$ NMR $\left(500 \mathrm{MHz}, \mathrm{DMSO}-\mathrm{d}_{6}\right) \mathrm{ppm} 6.93(2 \mathrm{H}$, $\left.\mathrm{d}, J=8.6 \mathrm{~Hz}, \mathrm{CH}, \mathrm{Ar}-\mathrm{H}_{3^{\prime}}\right) 7.58(2 \mathrm{H}, \mathrm{d}, J=8 \mathrm{~Hz}, \mathrm{CH}$, Ar $\left.-\mathrm{H}_{2}\right) 7.79\left(2 \mathrm{H}, \mathrm{d}, J=8.6 \mathrm{~Hz}, \mathrm{CH}, \mathrm{Ar}-\mathrm{H}_{2^{\prime}}\right) 7.92(2 \mathrm{H}, \mathrm{d}$, $\left.J=8 \mathrm{~Hz}, \mathrm{CH}, \mathrm{Ar}-\mathrm{H}_{3}\right) 10.41(1 \mathrm{H}, \mathrm{s}, \mathrm{OH}){ }^{13} \mathrm{C} \mathrm{NMR}(125 \mathrm{MHz}$, DMSO-d 6 ) ppm $97.45\left(\right.$ Ar- $\left.C_{4}\right), 116.07\left(\mathrm{Ar}_{-} \mathrm{C}_{3^{\prime}}\right), 124.06(\mathrm{Ar}-$ $\left.\mathrm{C}_{2^{\prime}}\right), 125.11\left(\mathrm{Ar}-\mathrm{C}_{2}\right), 138.29\left(\mathrm{Ar}-\mathrm{C}_{3}\right), 145.15\left(\mathrm{Ar}-\mathrm{C}_{1^{\prime}}\right), 151.46$ $\left(\mathrm{Ar}-\mathrm{C}_{1}\right), 161.32\left(\mathrm{Ar}-\mathrm{C}_{4^{\prime}}\right)$.

2.4. General Method for the Synthesis of Aspirin-Halogenated Azo Derivatives $\mathbf{2 a}$-d. Azo (1a) (0.65 g, $3 \mathrm{mmol}$ ) was dissolved in dry DCM $(20 \mathrm{~mL})$ and added to a round bottom flask containing acetylsalicylic acid $(0.54 \mathrm{~g}, 3 \mathrm{mmol})$ in dry DCM $(20 \mathrm{~mL})$ at $0-10^{\circ} \mathrm{C}$. DCC $(0.62 \mathrm{~g}, 3 \mathrm{mmol})$ and DMAP $(0.37 \mathrm{~g}, 3 \mathrm{mmol})$ were added to the mixture and stirred for $4 \mathrm{~h}$ at $0-10^{\circ} \mathrm{C}$. The mixture was filtered and evaporated. The crude was recrystallized from hot ethanol to give (2a) $(0.58 \mathrm{~g}, 51 \%)$ as pale yellow solid; m.p. $147-148^{\circ} \mathrm{C}$. (Found: C, 66.65; H, 3.96; $\mathrm{N}, 7.22 \% \mathrm{C}_{21} \mathrm{H}_{15} \mathrm{~N}_{2} \mathrm{O}_{4} \mathrm{~F}$ Requires C, 66.67; H, 3.97; N, 7.41\%); $R_{f} 0.62\left(1: 4\right.$ EA/hexane) IR: $v_{\max }\left(\right.$ thin film $\left./ \mathrm{cm}^{-1}\right) 1755,1745$ $(\mathrm{C}=\mathrm{O}), 1590(\mathrm{C}=\mathrm{C}$ aromatic), $1482(\mathrm{~N}=\mathrm{N}), 1222(\mathrm{C}-\mathrm{N}), 1184$ (C-O), $841(\mathrm{C}-\mathrm{F}){ }^{1} \mathrm{H}$ NMR $\left(500 \mathrm{MHz}, \mathrm{DMSO}-\mathrm{d}_{6}\right.$ ) ppm 2.27 $\left(3 \mathrm{H}, \mathrm{s},-\mathrm{CH}_{3}\right) 7.35\left(1 \mathrm{H}, \mathrm{d}, J=7.80 \mathrm{~Hz}, \mathrm{Ar}_{-} \mathrm{H}_{5^{\prime \prime}}\right) 7.44(2 \mathrm{H}, \mathrm{d}$, $\left.J=8.60 \mathrm{~Hz}, \mathrm{Ar}-\mathrm{H}_{3}\right) 7.47\left(2 \mathrm{H}, \mathrm{d}, J=8.60 \mathrm{~Hz}, \mathrm{Ar}-\mathrm{H}_{3^{\prime}}\right) 7.51$ $\left(1 \mathrm{H}, \mathrm{t}, J=7.55 \mathrm{~Hz}, \mathrm{Ar}-\mathrm{H}_{4^{\prime \prime}}\right) 7.79\left(1 \mathrm{H}, \mathrm{t}, J=7.8 \mathrm{~Hz}, \mathrm{Ar}-\mathrm{H}_{3^{\prime \prime}}\right)$ 7.98-8.01 (4H, m, Ar- $\left.\mathrm{H}_{2,2^{\prime}}\right) 8.20\left(1 \mathrm{H}, \mathrm{d}, J=7.80 \mathrm{~Hz}, \mathrm{Ar}-\mathrm{H}_{2^{\prime \prime}}\right)$ ${ }^{13} \mathrm{C}$ NMR $\left(125 \mathrm{MHz}, \mathrm{DMSO}-\mathrm{d}_{6}\right) 20.73\left(-\mathrm{CH}_{3}\right), 116.43(\mathrm{Ar}-$ $\left.\mathrm{C}_{3}\right), 116.61\left(\mathrm{Ar}-\mathrm{C}_{1^{\prime \prime}}\right), 121.98\left(\mathrm{Ar}-\mathrm{C}_{3^{\prime}}\right), 122.98\left(\mathrm{Ar}-\mathrm{C}_{2^{\prime}}\right), 123.98$ $\left(\mathrm{Ar}-\mathrm{C}_{5^{\prime \prime}}\right), 124.23\left(\mathrm{Ar}-\mathrm{C}_{2}\right), 125.03\left(\mathrm{Ar}-\mathrm{C}_{3^{\prime \prime}}\right), 126.57\left(\mathrm{Ar}-\mathrm{C}_{2^{\prime \prime}}\right)$, 131.87 $\left(\right.$ Ar- $\left._{4^{\prime \prime}}\right), 135.33\left(\right.$ Ar- $\left.\mathrm{C}_{1^{\prime}}\right), 148.57($ Ar-C 1 ), $149.70($ Ar$\left.\mathrm{C}_{6^{\prime \prime}}\right), 150.49\left(\mathrm{Ar}_{-} \mathrm{C}_{4^{\prime}}\right), 152.43\left(\mathrm{Ar}-\mathrm{C}_{4}\right), 162.41\left(\mathrm{C}_{\mathrm{a}}=\mathrm{O}\right), 169.24$ $\left(\mathrm{C}_{\mathrm{b}}=\mathrm{O}\right)$. The others, $\mathbf{2 b}-\mathbf{d}$, were synthesized following the same procedure as $\mathbf{2 a}$ with yields given below. 
4-[(E)-(Chlorophenyl)diazenyl]phenyl aspirinate (2b) $(0.54 \mathrm{~g}, 46 \%)$ as orange solid; m.p. $161-162^{\circ} \mathrm{C}$. (Found: C, 63.58; $\mathrm{H}, 3.73 \mathrm{~N}, 7.44 \% \mathrm{C}_{21} \mathrm{H}_{15} \mathrm{~N}_{2} \mathrm{O}_{4} \mathrm{Cl}$ Requires C, 63.88; $\mathrm{H}, 3.80 ; \mathrm{N}, 7.10 \%) ; R_{f} 0.64$ (1:4 EA/hexane) IR: $v_{\max }$ (thin film $\left./ \mathrm{cm}^{-1}\right) 1766,1740(\mathrm{C}=\mathrm{O}), 1588(\mathrm{C}=\mathrm{C}$ aromatic), $1479(\mathrm{~N}=\mathrm{N}), 1246(\mathrm{C}-\mathrm{N}), 1182(\mathrm{C}-\mathrm{O}), 833(\mathrm{C}-\mathrm{Cl}){ }^{1} \mathrm{H}$ NMR $\left(500 \mathrm{MHz}, \mathrm{DMSO}-\mathrm{d}_{6}\right)$ ppm $2.27\left(3 \mathrm{H}, \mathrm{s},-\mathrm{CH}_{3}\right) 7.35(1 \mathrm{H}, \mathrm{d}$, $\left.J=8.6 \mathrm{~Hz}, \mathrm{Ar}-\mathrm{H}_{5^{\prime \prime}}\right) 7.49\left(2 \mathrm{H}, \mathrm{d}, J=9.2 \mathrm{~Hz}, \mathrm{Ar}-\mathrm{H}_{3^{\prime}}\right) 7.53(\mathrm{H}$, $\left.\mathrm{t}, J=7.5 \mathrm{~Hz}, \mathrm{Ar}-\mathrm{H}_{4^{\prime \prime}}\right) 7.68\left(2 \mathrm{H}, \mathrm{d}, J=8.6 \mathrm{~Hz}, \mathrm{Ar}-\mathrm{H}_{3}\right) 7.81$ $\left(1 \mathrm{H}, \mathrm{t}, J=7.7 \mathrm{~Hz}, \mathrm{Ar}-\mathrm{H}_{3^{\prime \prime}}\right) 7.93\left(2 \mathrm{H}, \mathrm{d}, J=9.2 \mathrm{~Hz}, \mathrm{Ar}-\mathrm{H}_{2}\right) 8.01$ $\left(2 \mathrm{H}, \mathrm{d}, J=8.6 \mathrm{~Hz}, \mathrm{Ar}-\mathrm{H}_{2^{\prime}}\right) 8.20\left(1 \mathrm{H}, \mathrm{d}, J=8 \mathrm{~Hz}, \mathrm{Ar}-\mathrm{H}_{2^{\prime \prime}}\right){ }^{13} \mathrm{C}$ NMR $\left(125 \mathrm{MHz}, \mathrm{DMSO}-\mathrm{d}_{6}\right) 20.74\left(-\mathrm{CH}_{3}\right), 121.96\left(\mathrm{Ar}-\mathrm{C}_{1^{\prime \prime}}\right)$, $123.04\left(\mathrm{Ar}^{-\mathrm{C}_{3^{\prime}}}\right), 124.14\left(\mathrm{Ar}-\mathrm{C}_{2^{\prime}}\right), 124.23\left(\mathrm{Ar}-\mathrm{C}_{2}\right), 124.31$ (Ar$\left.\mathrm{C}_{5^{\prime \prime}}\right), 126.57\left(\mathrm{Ar}-\mathrm{C}_{3^{\prime \prime}}\right), 128.78\left(\mathrm{Ar}-\mathrm{C}_{3}\right), 129.67\left(\mathrm{Ar}-\mathrm{C}_{2^{\prime \prime}}\right), 131.88$ $\left(\mathrm{Ar}-\mathrm{C}_{4^{\prime \prime}}\right), 134.99\left(\mathrm{Ar}-\mathrm{C}_{4}\right), 136.09\left(\mathrm{Ar} \mathrm{C}_{1^{\prime}}\right), 149.71\left(\mathrm{Ar}-\mathrm{C}_{1}\right)$, $150.50\left(\right.$ Ar- $\left._{6^{\prime \prime}}\right), 152.50\left(\right.$ Ar- $\left._{4^{\prime}}\right), 162.38\left(\mathrm{C}_{\mathrm{a}}=\mathrm{O}\right), 169.35$ $\left(\mathrm{C}_{\mathrm{b}}=\mathrm{O}\right)$.

4-[(E)-(Bromophenyl)diazenyl]phenyl aspirinate (2c) $(0.59 \mathrm{~g}, 45 \%)$ as yellow solid; m.p. $149-151^{\circ} \mathrm{C}$. (Found: C, 57.31; $\mathrm{H}, 3.39 ; \mathrm{N}, 6.02 \% \mathrm{C}_{21} \mathrm{H}_{15} \mathrm{~N}_{2} \mathrm{O}_{4} \mathrm{Br}$ Requires C, 57.40; $\mathrm{H}, 3.42 ; \mathrm{N}, 6.38 \%) ; R_{f} 0.62$ (1:4 EA/hexane) IR: $v_{\max }$ (thin film $\left./ \mathrm{cm}^{-1}\right) 1763,1734(\mathrm{C}=\mathrm{O}), 1581(\mathrm{C}=\mathrm{C}$ aromatic), $1477(\mathrm{~N}=\mathrm{N}), 1250(\mathrm{C}-\mathrm{N}), 1179(\mathrm{C}-\mathrm{O}), 829(\mathrm{C}-\mathrm{Br}){ }^{1} \mathrm{H}$ NMR $\left(500 \mathrm{MHz}, \mathrm{DMSO}-\mathrm{d}_{6}\right)$ ppm $2.27\left(3 \mathrm{H}, \mathrm{s},-\mathrm{CH}_{3}\right) 7.34(1 \mathrm{H}, \mathrm{d}$, $\left.J=8.6 \mathrm{~Hz}, \mathrm{Ar}-\mathrm{H}_{5^{\prime \prime}}\right) 7.48\left(2 \mathrm{H}, \mathrm{d}, J=9.15 \mathrm{~Hz}, \mathrm{Ar}-\mathrm{H}_{3^{\prime}}\right) 7.51(1 \mathrm{H}$, $\left.\mathrm{t}, J=8.3 \mathrm{~Hz}, \mathrm{Ar}-\mathrm{H}_{4^{\prime \prime}}\right) 7.79-7.87\left(5 \mathrm{H}, \mathrm{m}, \mathrm{Ar}-\mathrm{H}_{2,3,3^{\prime \prime}}\right) 8.01(2 \mathrm{H}$, d, $\left.J=6.9 \mathrm{~Hz}, \mathrm{Ar}-\mathrm{H}_{2^{\prime}}\right) 8.20\left(1 \mathrm{H}, \mathrm{d}, J=9.15 \mathrm{~Hz}, \mathrm{Ar}-\mathrm{H}_{2^{\prime \prime}}\right){ }^{13} \mathrm{C}$ NMR $\left(125 \mathrm{MHz}, \mathrm{DMSO}-\mathrm{d}_{6}\right) 20.78\left(-\mathrm{CH}_{3}\right), 121.91\left(\mathrm{Ar}-\mathrm{C}_{1^{\prime \prime}}\right)$, $123.09\left(\mathrm{Ar}^{-\mathrm{C}_{3^{\prime}}}\right), 124.20\left(\mathrm{Ar}-\mathrm{C}_{2^{\prime}}\right), 124.26\left(\mathrm{Ar}-\mathrm{C}_{5^{\prime \prime}}\right), 124.55(\mathrm{Ar}-$ $\left.\mathrm{C}_{2}\right), 125.14\left(\mathrm{Ar}^{-\mathrm{C}_{4}}\right), 126.62\left(\mathrm{Ar}-\mathrm{C}_{3^{\prime \prime}}\right), 131.92\left(\mathrm{Ar}-\mathrm{C}_{2^{\prime \prime}}\right), 132.66$ $\left(\mathrm{Ar}-\mathrm{C}_{3}\right), 135.34\left(\mathrm{Ar}-\mathrm{C}_{4^{\prime \prime}}\right), 149.67\left(\mathrm{Ar}-\mathrm{C}_{1^{\prime}}\right), 150.12\left(\mathrm{Ar}-\mathrm{C}_{6^{\prime \prime}}\right)$,

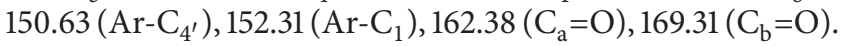

3-[(E)-(Iodophenyl)diazenyl]phenyl aspirinate (2d) $(0.79 \mathrm{~g}, 54 \%)$ as orange solid; m.p. $180-181^{\circ} \mathrm{C}$. (Found: C, 51.63; H, 3.06; N, 5.43\% $\mathrm{C}_{21} \mathrm{H}_{15} \mathrm{~N}_{2} \mathrm{O}_{4} \mathrm{I}$ Requires $\mathrm{C}$, 51.85; $\mathrm{H}, 3.09 ; \mathrm{N}, 5.76 \%) ; R_{f} 0.63(1: 4 \mathrm{EA} /$ hexane $)$ IR: $v_{\max }$ (thin film $\left./ \mathrm{cm}^{-1}\right) 1764,1732(\mathrm{C}=\mathrm{O}), 1578(\mathrm{C}=\mathrm{C}$ aromatic), $1475(\mathrm{~N}=\mathrm{N}), 1250(\mathrm{C}-\mathrm{N}), 1176(\mathrm{C}-\mathrm{O}), 826(\mathrm{C}-\mathrm{I}){ }^{1} \mathrm{H}$ NMR $\left(500 \mathrm{MHz}, \mathrm{DMSO}-\mathrm{d}_{6}\right) 2.27\left(3 \mathrm{H}, \mathrm{s},-\mathrm{CH}_{3}\right) \mathrm{ppm} 7.35(1 \mathrm{H}, \mathrm{d}$, $\left.J=8.0 \mathrm{~Hz}, \mathrm{Ar}-\mathrm{H}_{5^{\prime \prime}}\right) 7.48\left(2 \mathrm{H}, \mathrm{d}, J=8.60 \mathrm{~Hz}, \mathrm{Ar}-\mathrm{H}_{3^{\prime}}\right) 7.51(1 \mathrm{H}$, t, $\left.J=7.45 \mathrm{~Hz}, \mathrm{Ar}-\mathrm{H}_{4^{\prime \prime}}\right) 7.69\left(2 \mathrm{H}, \mathrm{d}, J=8.0 \mathrm{~Hz}, \mathrm{Ar}-\mathrm{H}_{2}\right) 7.79$ $\left(1 \mathrm{H}, \mathrm{t}, J=8.0 \mathrm{~Hz}, \mathrm{Ar}-\mathrm{H}_{3^{\prime \prime}}\right) .7 .99-8.03\left(4 \mathrm{H}, \mathrm{m}, \mathrm{Ar}-\mathrm{H}_{3,2^{\prime}}\right) 8.20$ $\left(1 \mathrm{H}, \mathrm{d}, J=8.0 \mathrm{~Hz}, \mathrm{Ar}-\mathrm{H}_{2^{\prime \prime}}\right){ }^{13} \mathrm{C}$ NMR $\left(125 \mathrm{MHz}\right.$, DMSO- $\left.\mathrm{d}_{6}\right)$ $20.78\left(-\mathrm{CH}_{3}\right), 99.40\left(\mathrm{Ar}-\mathrm{C}_{4}\right), 121.96\left(\mathrm{Ar}-\mathrm{C}_{1^{\prime \prime}}\right), 123.08(\mathrm{Ar}-$ $\left.\mathrm{C}_{3^{\prime}}\right), 124.18\left(\mathrm{Ar}-\mathrm{C}_{2^{\prime}}\right), 124.48\left(\mathrm{Ar}-\mathrm{C}_{5^{\prime \prime}}\right), 126.62\left(\mathrm{Ar}-\mathrm{C}_{2}\right), 131.92$ $\left(\mathrm{Ar}-\mathrm{C}_{3^{\prime \prime}}\right), 135.33\left(\mathrm{Ar}-\mathrm{C}_{2^{\prime \prime}}\right), 137.56\left(\mathrm{Ar}-\mathrm{C}_{4^{\prime \prime}}\right), 138.54\left(\mathrm{Ar}-\mathrm{C}_{3}\right)$, $149.60\left(\mathrm{Ar}^{-\mathrm{C}_{1^{\prime}}}\right), 150.43\left(\mathrm{Ar}-\mathrm{C}_{6^{\prime \prime}}\right), 151.11\left(\mathrm{Ar}-\mathrm{C}_{4^{\prime}}\right), 152.72$ $\left(\operatorname{Ar}-\mathrm{C}_{1}\right), 162.21\left(\mathrm{C}_{\mathrm{a}}=\mathrm{O}\right), 169.17\left(\mathrm{C}_{\mathrm{b}}=\mathrm{O}\right)$.

2.5. Cell Culture. The cell viability of nasopharyngeal cancer cell lines HK-1 was determined by MTS-based colorimetric assay. The cells were kept at $37^{\circ} \mathrm{C}$ in a $5 \% \mathrm{CO}_{2}$ in RPMI 1640 (GIBCO) supplemented with $10 \%$ fetal bovine serum (FBS; Sigma-Aldrich). The NPC cells were assayed with a volume of $100 \mu \mathrm{L}$ to each well plate at a concentration of 5000 cells/well. The drug dissolved in DMSO and a compound dilution 96well plate was prepared at different concentrations of which $100 \mu \mathrm{L} /$ well of the diluted mixtures was then added. A volume of $0.5 \mu \mathrm{L}(0.1 \% \mathrm{DMSO})$ was added with cells as a control. The final drug concentrations were ranging from 0.39 to $50 \mu \mathrm{M}$ at $200 \mu \mathrm{L}$. The cells were incubated for $72 \mathrm{~h}$ at $37^{\circ} \mathrm{C}$. After incubation, the media were discarded from each well and added with $10 \mu \mathrm{L}$ MTS solution and $50 \mu \mathrm{L}$ fresh media. The plates were further incubated for 30 minutes at $37^{\circ} \mathrm{C}$. The absorbance was measured by a multiwell plate reader at $490 \mathrm{~nm}$. This experiment was performed in triplicate [22].

2.6. Statistical Analyses. The quantitative data are expressed as mean \pm standard deviation. The cell viability against $\log \mathrm{IC}_{50}$ values of all compounds was determined using Graphpad prism 6 program.

\section{Results and Discussion}

The synthesis of 4-[(halophenyl)diazenyl]phenyl aspirinate (2a-d) was carried out via esterification of aspirin and 4-[(halophenyl)diazenyl]phenol $(\mathbf{l a}-\mathbf{d})$ in the presence of DCC/DMAP in DCM. 4-[(Halophenyl)diazenyl]phenol (1a-d) was earlier synthesized from the reaction of aniline derivatives and phenol via direct coupling reaction. The synthesis of 2a-d is depicted in Scheme 1. Characteristic spectroscopic data of the synthesised compounds are provided in the supplementary data in Supplementary Material available online at https://doi.org/10.1155/2017/6760413.

All compounds were characterized using CHN, FTIR, and ${ }^{1} \mathrm{H}$ and ${ }^{13} \mathrm{C}$ NMR spectroscopy. FTIR spectra of $\mathbf{l a}-\mathbf{d}$ and $2 \mathrm{a}-\mathbf{d}$ showed the absorption peaks at $1474-1471 \mathrm{~cm}^{-1}$ and $1591-1587 \mathrm{~cm}^{-1}$ attributed to $v(-\mathrm{N}=\mathrm{N}-)$ and $v(\mathrm{C}=\mathrm{C})$, respectively. The formation of $\mathbf{2 a - d}$ was confirmed by the disappearance of $v(\mathrm{OH})$ peak at $3186-3076 \mathrm{~cm}^{-1}$ and the appearance of new absorption peaks at $1765-1745 \mathrm{~cm}^{-1}$ corresponded to $v(\mathrm{C}=\mathrm{O})$ ester. The absorption frequencies at $841-826 \mathrm{~cm}^{-1}$ in both FTIR spectra of $1 \mathbf{a}-\mathbf{d}$ and $\mathbf{2 a - d}$ corresponded to para-halogenated substitution [23].

${ }^{1} \mathrm{H}$ NMR spectra of $\mathbf{1 a}-\mathbf{d}$ and $\mathbf{2 a}-\mathbf{d}$ showed the presence of aromatic protons at $8.20-6.93 \mathrm{ppm}$, while $\mathrm{CH}_{3}$ in $\mathbf{2 a - d}$ was observed at $2.27 \mathrm{ppm} .{ }^{13} \mathrm{C}$ NMR spectra of $1 \mathbf{a}-\mathbf{d}$ and $2 \mathbf{a}-\mathbf{d}$ showed resonance of aromatic carbons at 152.7-99.4 ppm. The presence of $\mathrm{C}=\mathrm{O}$ and $\mathrm{CH}_{3}$ in $\mathbf{2 a}-\mathbf{d}$ was observed at 169.4-162.4 ppm and 20.8-20.7 ppm, respectively.

3.1. Anticancer Activity. Compounds 1a-d and 2a-d were screened for anticancer activity against NPC HK-1 cell lines. The anticancer activities of $\mathbf{1 a}-\mathbf{d}$ and $\mathbf{2 a - d}$ on NPC HK1 cell lines were studied by MTS based on colorimetric assay. The cells were seeded onto 96-well culture plates and incubated with different concentrations of compounds (up to $50 \mu \mathrm{M}$ ) for $72 \mathrm{~h}$. The relative influence of $\mathbf{l a}-\mathbf{d}$ and $2 \mathbf{a}-\mathbf{d}$ was determined via viability of the NPC cell line against $\log$ [concentration, $\mu \mathrm{M}$ ] as shown in Figure 1 . The $\mathrm{IC}_{50}$ of $\mathbf{1 a}-\mathbf{d}$ and $\mathbf{2 a}-\mathbf{d}$ is summarized in Table 1. Among four halogen substituents of 1a-d, la substituted with fluorine showed good anticancer activity against HK-1 cell lines with $\mathrm{IC}_{50}$ of $19.1 \pm 0.9 \mu \mathrm{M}$. In comparison to $\mathrm{IC}_{50}$ of positive control (standard drug), 5-fluorouracil, and cisplatin with $12.5 \pm 1.4 \mu \mathrm{M}$ and $8.9 \pm 1.9 \mu \mathrm{M}$, respectively, $\mathrm{IC}_{50}$ of $\mathbf{1 a}$ 


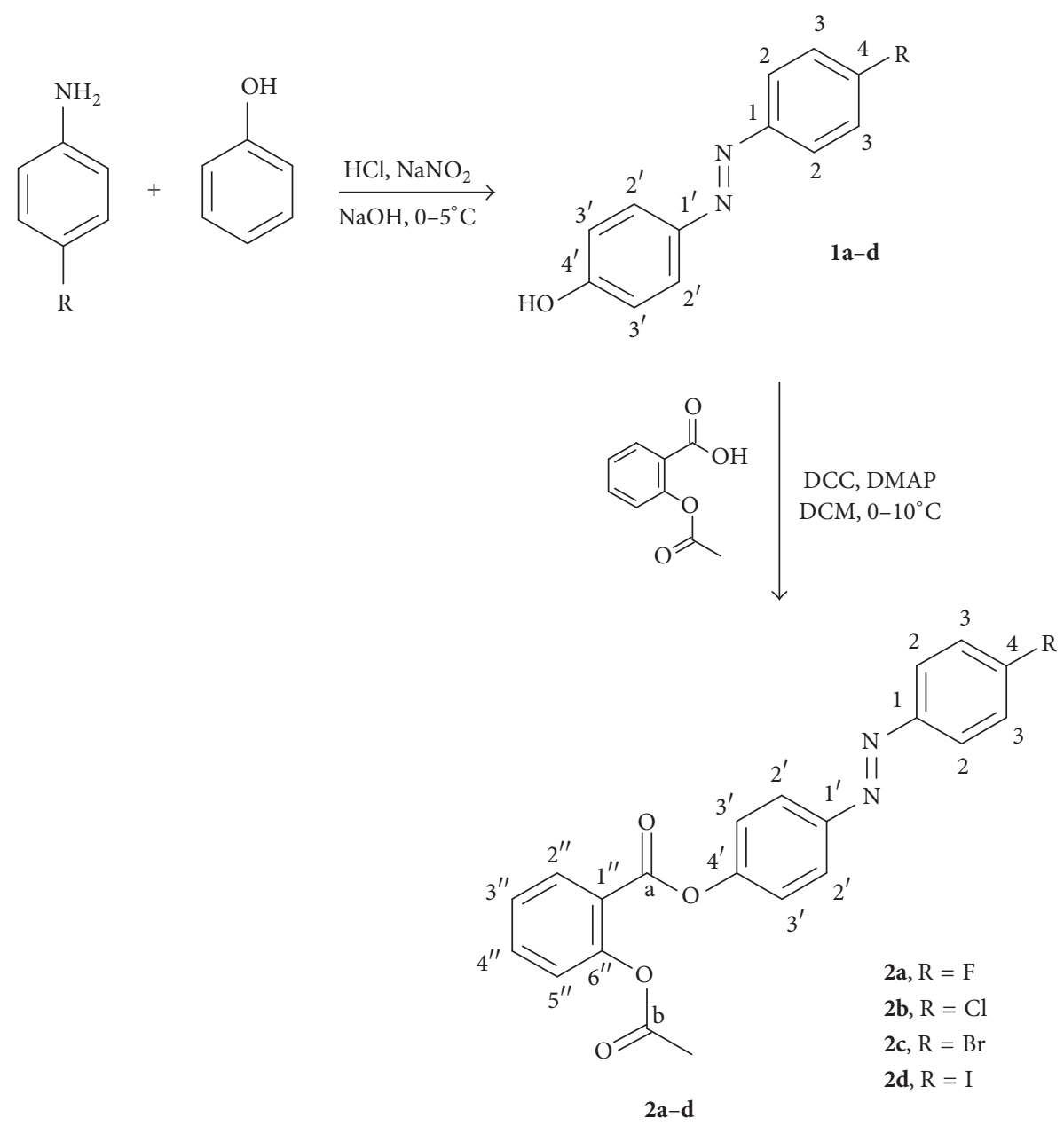

Scheme 1: Synthesis of $\mathbf{1 a - d}$ and $\mathbf{2 a - d .}$

was comparable to 5 -fluorouracil. Compound la-d bearing halogen substituents has good lipophilic property and thus improved the binding properties towards the active site of cells enzyme [21,24]. Among all halogens, fluorine is the most selectively bound halogen to specific enzyme and remains covalently and irreversibly bound to the active site of enzyme, hence providing good anticancer activity [25]. In addition, the presence of $-\mathrm{N}=\mathrm{N}$ - group in the molecular structure has also contributed to anticancer activity by interaction with the active site of protein via hydrogen bonding resulting in the inhibition growth of cells [20]. The presence of phenyl ring is also important to provide more lipophilic character [26]. Compound with high lipophilicity has potential to inhibit proteins for cell growth [27].

Anticancer activity of $\mathbf{2 a - d}$ against NPC HK-1 cell lines only showed $2 \mathbf{a}(38.7 \pm 0.8 \mu \mathrm{M})$ and $2 \mathbf{d}(44.9 \pm 5.4 \mu \mathrm{M})$ with average anticancer activity compared to the aspirin alone. The presence of azo moiety in the molecular structure has contributed to the anticancer activity [20]. The weak inhibition on NPC HK-1 cell lines on $\mathbf{2 c - 2 d}$ was due to higher molecular weight with larger size of bromine and
TABLE 1: $\mathrm{IC}_{50}$ of 1a-d and 2a-d, aspirin, 5-fluorouracil, and cisplatin against NPC HK-1 cells lines.

\begin{tabular}{lc}
\hline Compound & $\mathrm{IC}_{50}(\mu \mathrm{M})$ \\
\hline Aspirin & $>50$ \\
cisplatin & $8.9 \pm 3.2$ \\
5-Fluorouracil & $12.5 \pm 2.5$ \\
la & $19.1 \pm 1.5$ \\
lb & $36.7 \pm 8.2$ \\
lc & $21.9 \pm 7.3$ \\
ld & $20.8 \pm 6.6$ \\
2a & $38.7 \pm 1.5$ \\
2b & $44.9 \pm 9.4$ \\
2c & $>50$ \\
2d & $>50$ \\
\hline
\end{tabular}

iodine atoms resulting in bulky molecules [28] and gave low cytotoxicity towards cancer cells. High molecular weight 

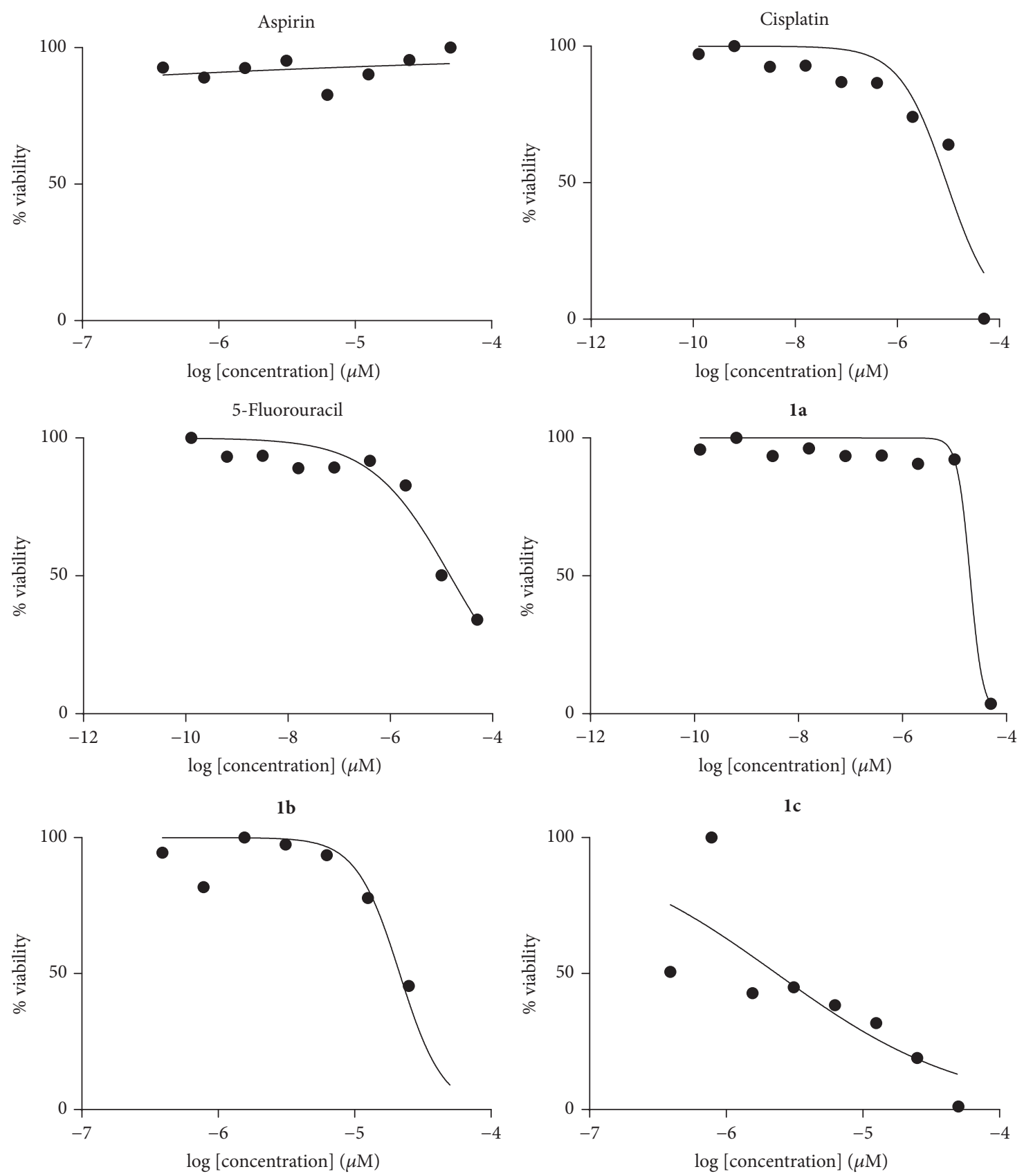

ld
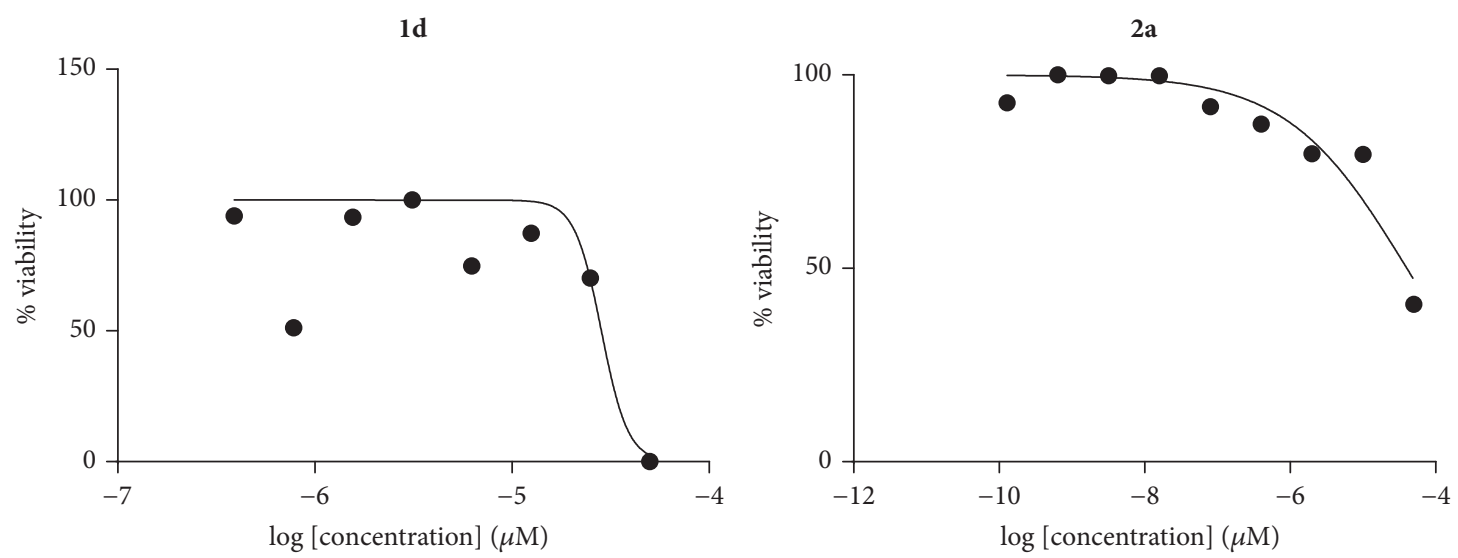

FIgure 1: Continued. 



FIGURE 1: Cell viability of NPC cells, HK1 treated with 1a-d and 2a-d, aspirin, and standards (cisplatin and 5-fluorouracil).

and bulky molecules contributed to steric hindrance, thus preventing $2 \mathbf{a}-\mathbf{d}$ from reaching the active site of enzyme [29].

\section{Conclusion}

In conclusion, a series of $\mathbf{1 a - d}$ and $\mathbf{2 a - d}$ were synthesized and screened for their anticancer activities based on MTS assay. Compound la-d demonstrated good anticancer activities compared to 2a-d, where la exhibited the highest cytotoxicity against NPC HK-1 cells lines, comparable to the standard drug (5-fluorouracil). These results indicated that $\mathbf{l a}-\mathbf{d}$ could be used as potential lead compounds for anticancer drug development. Compounds 2a-d provide low cytotoxicity due to the occurrence of steric hindrance which prevent them from reaching the receptor of enzymes.

\section{Conflicts of Interest}

The authors declare that they have no conflicts of interest.

\section{Acknowledgments}

The authors wish to acknowledge the University Malaysia Sarawak and Ministry of Higher Education for financial support of this project through Grants FRGS/ST02(01)/1115/ 2014(01) and FRGS/ST0l(01)/1298/2015(15).

\section{References}

[1] "World Health Organization. Health at a glance: Asia/Pacific 2014: Measuring progress towards universal health coverage, Paris, OECD Publishing, 2014".

[2] G. M. Cooper, The cell: A molecular approach, Sinauer Associates, Sunderland, UK, 2nd edition, 2000.

[3] B.-J. Feng, M. Jalbout, W. B. Ayoub et al., "Dietary risk factors for nasopharyngeal carcinoma in Maghrebian countries," International Journal of Cancer, vol. 121, no. 7, pp. 1550-1555, 2007.

[4] E. T. Chang and H. Adami, "The enigmatic epidemiology of nasopharyngeal carcinoma," Cancer Epidemiology Biomarkers and Prevention, vol. 15, no. 10, pp. 1765-1777, 2006.

[5] F. L. Sung, T. C. W. Poon, and E. P. Hui, "Antitumor effect and enhancement of cytotoxic drug activity by cetuximab in nasopharyngeal carcinoma cells," In Vivo, vol. 19, no. 1, pp. 237246, 2005.

[6] P. M. Harari, "Epidermal growth factor receptor inhibition strategies in oncology," Endocrine-Related Cancer, vol. 11, no. 4, pp. 689-708, 2004.

[7] M. Gleeson, G. G. Browning, M. J. Burton et al., Scott-Browns otorhinolaryngology, head and neck surgery, Hodder Arnold, London, UK, 7th edition, 2008.

[8] Y. C. Mayur, G. J. Peters, V. V. S. Rajendra Prasad, C. Lemos, and N. K. Sathish, "Design of new drug molecules to be used in reversing multidrug resistance in cancer cells," Current Cancer Drug Targets, vol. 9, no. 3, pp. 298-306, 2009. 
[9] A. I. Francisco, M. D. Vargas, T. P. Fragoso et al., "Theoretical studies of the tautomerism in 3-(2-R-phenylhydrazono)naphthalene-1,2,4-triones: Synthesis of copper(II) complexes and studies of antibacterial and antitumor activities," Journal of the Brazilian Chemical Society, vol. 21, no. 7, pp. 1293-1302, 2010.

[10] L. Huang, G. G. MacKenzie, N. Ouyang et al., "The novel phospho-non-steroidal anti-inflammatory drugs, OXT-328, MDC22 and MDC-917, inhibit adjuvant-induced arthritis in rats," British Journal of Pharmacology, vol. 162, no. 7, pp. 1521-1533, 2011.

[11] N. A. Nordin, T. W. Chai, B. L. Tan et al., "Novel synthetic monothiourea aspirin derivatives bearing alkylated amines as potential antimicrobial agents," Journal of Chemistry, vol. 2017, pp. 1-7, 2017.

[12] Z. Ngaini, M. A. Mohd Arif, H. Hussain, E. S. Mei, D. Tang, and D. H. A. Kamaluddin, "Synthesis and antibacterial activity of acetoxybenzoyl thioureas with aryl and amino acid side Chains," Phosphorus, Sulfur, and Silicon and the Related Elements, vol. 187, no. 1, pp. 1-7, 2012.

[13] K. Kashfi and B. Rigas, "The mechanism of action of nitric oxide-donating aspirin," Biochemical and Biophysical Research Communications, vol. 35, no. 8, pp. 1096-1101, 2007.

[14] A. Cemel and S. Ahmet, "Rapid and simultaneous determination of acetylsalicylic acid, paracetamol and their degradation and toxic impurity product by HPLC in pharmaceutical dosage forms," Turkish Journals Medical Science, vol. 38, pp. 167-173, 2008.

[15] H. G. Garg and C. Praksh, "Preparation of 4-arylazo-3,5-disubstituted-(2H)-1,2,6-thiadiazine 1,1-dioxides," Journal Medicinal Chemistry, vol. 15, no. 4, pp. 435-439, 1972.

[16] R. G. Child, R. G. Wilkinson, and A. Tomcu-Fucik, "Effect of substrate orientation of the adhesion of polymer joints," Journal of Chemical Society, vol. 87, pp. 6031-6038, 1977.

[17] A. Khalid, M. Arshad, and D. E. Crowley, "Accelerated decolorization of structurally different azo dyes by newly isolated bacterial strains," Applied Microbiology and Biotechnology, vol. 78, no. 2, pp. 361-369, 2008.

[18] T. A. Farghaly and Z. A. Abdallah, "Synthesis, azo-hydrazone tautomerism and antitumor screening of $\mathrm{N}$-(3-ethoxycarbonyl4,5,6,7-tetrahydro-benzo[b]thien-2-yl)-2-arylhydrazono-3- oxobutanamide derivatives," Archieve for Organic Chemistry, vol. 17, no. 17, pp. 295-305, 2008.

[19] C. Park, J.-S. Lim, Y. Lee et al., "Optimization and morphology for decolorization of reactive black 5 by Funalia trogii," Enzyme Microbial Technology, vol. 40, no. 7, pp. 1758-1764, 2007.

[20] N. Raman, L. Mitu, A. Sakthivel, and M. S. S. Pandi, "Studies on DNA cleavage and antimicrobial screening of transition metal complexes of 4-aminoantipyrine derivatives of $\mathrm{N}_{2} \mathrm{O}_{2}$, Journal of the Iranian Chemical Society, vol. 6, no. 4, pp. 738-748, 2009.

[21] M. Z. Hernandes, S. M. T. Cavalcanti, D. R. M. Moreira, W. F. De Azevedo Jr., and A. C. L. Leite, "Halogen atoms in the modern medicinal chemistry: hints for the drug design," Current Drug Targets, vol. 11, no. 3, pp. 303-314, 2010.

[22] G. K. Y. Chan, T. L. Kleinheinz, D. Peterson, and J. G. Moffat, "A simple high-content cell cycle assay reveals frequent discrepancies between cell number and ATP and MTS proliferation assays," PLoS ONE, vol. 8, no. 5, Article ID e63583, 2013.

[23] T. W. G. Solomons and C. B. Fryhle, Organic Chemistry, John Wiley Sons, 8th edition, 2003.

[24] N. R. Modi, R. J. Shah, M. J. Patel, M. Suthar, B. F. Chauhan, and L. J. Patel, "Design, synthesis, and QSAR study of novel
2-(2, 3-dioxo-2, 3-dihydro-1H-indol-1-yl)-N-phenyl acetamide derivatives as cytotoxic agents," Medicinal Chemistry Research, vol. 20, pp. 615-625, 2011.

[25] J. Zhang, P. L. Yang, and N. S. Gray, “Targeting cancer with small molecule kinase inhibitors," Nature Review Cancer, vol. 9, no. 1, pp. 28-39, 2009.

[26] A. J. Hoey, C. M. Jackson, G. G. Pegg, and M. N. Sillence, "Characteristics of cyanopindolol analogues active at the beta(3)-adrenoceptor in rat ileum," British Journal of Pharmacology, vol. 119 , no. 3, pp. 564-568, 1996.

[27] J. C. Lopez-Romero, H. Gonzalez-Rios, A. Borges, and M. Simoes, "Antibacterial effects and mode of action of selected essential oils components against Escherichia coli and Staphylococcus aureus," Evidence-Based Complementary and Alternative Medicine, vol. 2015, Article ID 795435, 9 pages, 2015.

[28] M. Hum, B. E. McLaughlin, G. Roman, J. Z. Vlahakis, W. A. Szarek, and K. Nakatsu, "The effects of azole-based heme oxygenase inhibitors on rat cytochromes P450 2E1 and 3A1/2 and human cytochromes P450 3A4 and 2D6," Journal of Pharmacology and Experimental Therapeutics, vol. 334, no. 3, pp. 981-987, 2010.

[29] C. H. Yun, K. E. Mengwasser, A. V. Toms, M. S. Woo, and G. Hetal, "The T790M mutation in EGFR kinase causes drug resistance by increasing the affinity for ATP," in Proceedings of the National Academy of Sciences, vol. 105, pp. 2070-2075, 2008. 



Carbohydrate Chemistry

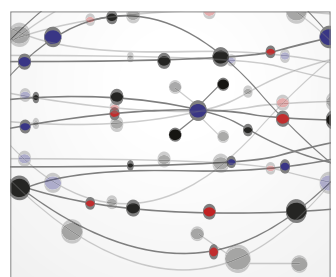

The Scientific World Journal
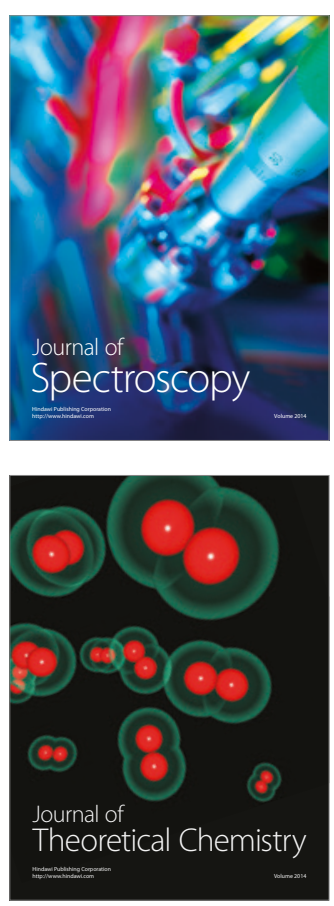
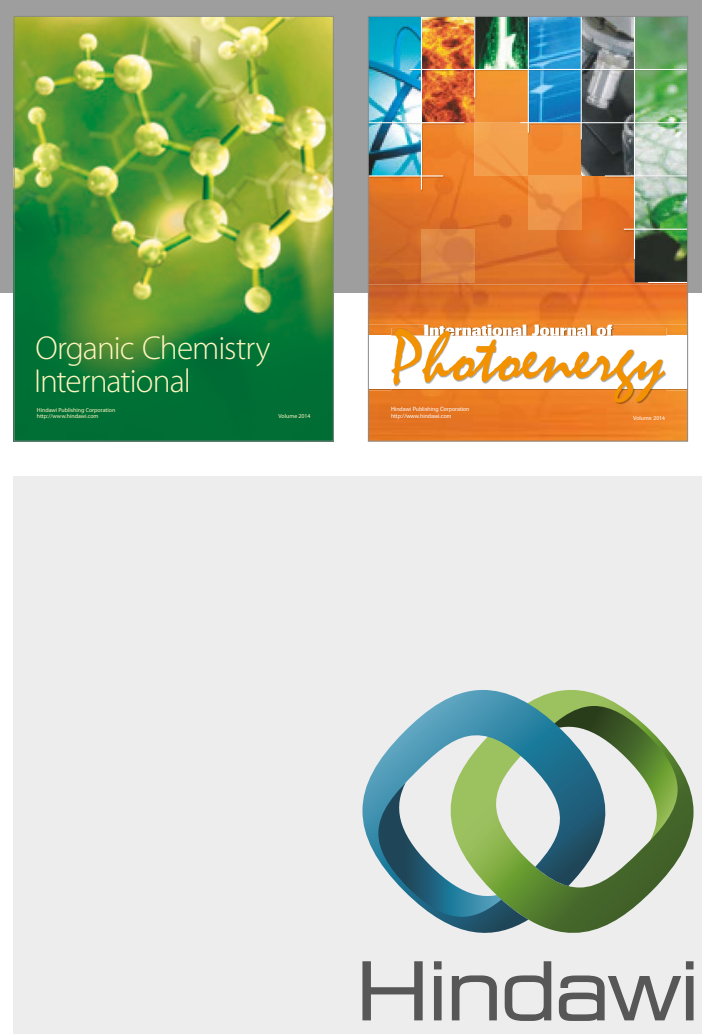

Submit your manuscripts at

https://www.hindawi.com

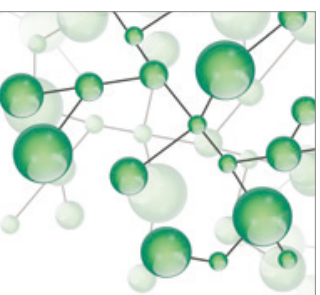

International Journal of

Inorganic Chemistry



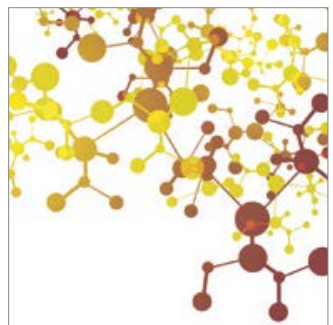

Applied Chemistry

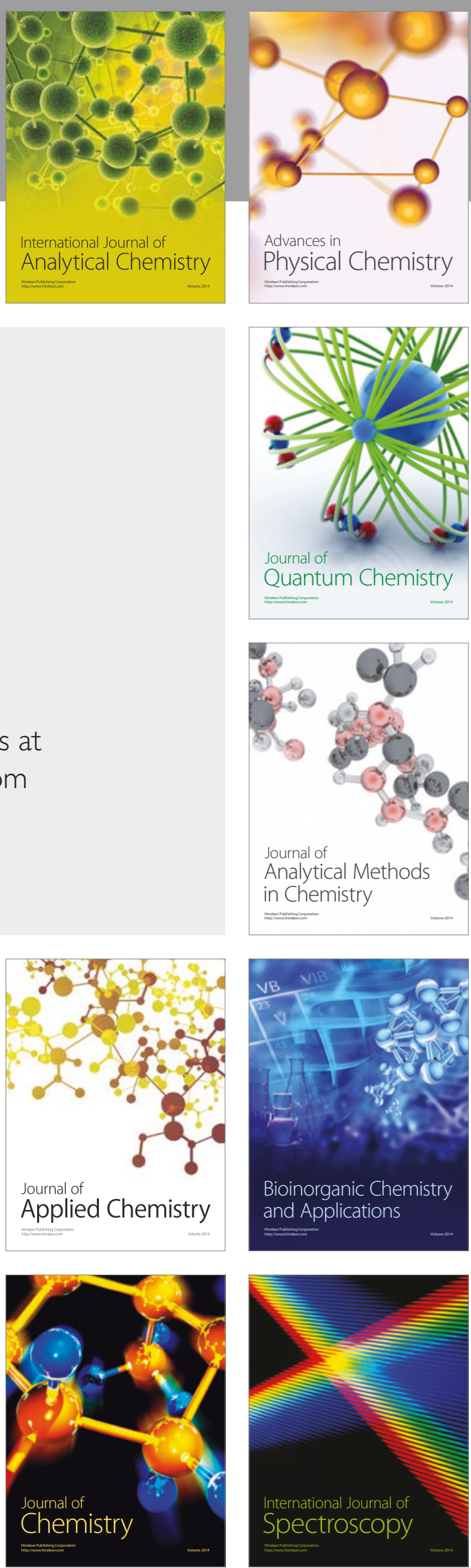